\title{
Eya2 overexpression promotes the invasion of human astrocytoma through the regulation of ERK/MMP9 signaling
}

\author{
ZHIFENG WEN, CHUANSHENG LIANG, QICHEN PAN and YUNJIE WANG \\ Department of Neurosurgery, The First Affiliated Hospital of \\ China Medical University, Shenyang, Liaoning 110001, P.R. China
}

Received October 16, 2016; Accepted August 14, 2017

DOI: $10.3892 /$ ijmm.2017.3132

\begin{abstract}
The overexpression of eyes absent (Eya) 2 has been found in several human cancers. However, its biological roles and clinical significance in human astrocytoma have not yet been explored. This study investigated the clinical significance and biological roles of Eya 2 in human astrocytoma tissues and cell lines. Using immunohistochemistry, we found Eya2 overexpression in 33 out of 90 (36.7\%) astrocytoma specimens. The rate of Eya2 overexpression was higher in grade III-IV (48.1\%) than in grade I+II astrocytomas $(21.1 \%)$ Transfection with an Eya2 expression plasmid was performed in A172 cells with a low endogenous expression of Eya2 and the knockdown of Eya2 was carried out in U251 cells with a high endogenous expression using siRNA. Eya2 overexpression induced A172 cell proliferation and invasion, while the knockdown of Eya2 using siRNA decreased the proliferation and invasion of U251 cells. In addition, we found that transfection with the Eya2 expression plasmid facilitated cell cycle progression, and that the knockdown of Eya2 inhibited cell cycle progression, accompanied by a change in the expression of cell cycle-related proteins, including cyclin D1 and cyclin E. Eya2 also positively regulated extracellular signal-regulated kinase (ERK) activity and matrix metalloproteinase (MMP)9 expression. The blockade of ERK signaling using an inhibitor abolished the effects of Eya2 on A172 cell invasion and MMP9 production. In addition, we found that there was a positive correlation between Eya2 and Six1 in the astrocytoma cell lines. Immunoprecipitation revealed that Eya2 interacted with Six1 protein in the U251 cell line, which exhibited a high expression of both proteins. Eya2 failed to upregulate MMP expression in the A172 cells in which Six1 was silenced. On the whole, our data indicate that Eya2 may serve as a potential oncoprotein in human astrocytoma. Eya2 regulates astro-
\end{abstract}

Correspondence to: Professor Yunjie Wang, Department of Neurosurgery, The First Affiliated Hospital of China Medical University, 155 Nanjing Street, Shenyang, Liaoning 110001, P.R. China E-mail:wangyj_neuro@yeah.net

Key words: Eya2, immunohistochemistry, astrocytoma, ERK cytoma cell proliferation and invasion, possibly through the regulation of ERK signaling.

\section{Introduction}

Astrocytoma is one of the most malignant tumors worldwide and its incidence is increasing. Despite progress being made in diagnosis and treatment over the past decades, its prognosis remains poor (1). The balance between tumor suppressors and oncogenes is very important during carcinogenesis and the progression of this malignant disease. Finding novel oncogenes which play an important role in its development would be helpful for the development of novel therapeutics $(2,3)$.

The MAPK/ERK signaling pathway is a highly conserved intracellular pathway that plays vital roles in the transmission of signals to the cell nucleus; these signals then transcriptionally regulate genes that are involved in various cellular processes, including cell invasion and metastasis (4-6). The invasion and metastasis of tumor cells, and the related degradation of the extracellular matrix (ECM) are closely associated with the development of malignant tumors. ECM degradation by extracellular proteinases is a key step and matrix metalloproteinase (MMPs) are primarily responsible for ECM degradation. In particular, MMP9 has been shown to play critical roles in the invasion and metastasis of many tumors (7-9) apart from astrocytoma.

The phosphatase and transactivator eyes absent (Eya) family encodes a group of transcription cofactors for eye development, which play important roles during organ development through multiple signaling pathways, including the epidermal growth factor receptor (EGFR), transforming growth factor (TGF), Hedgehog and Notch pathways (10-12). Recent studies have indicated their roles in human cancer proliferation and metastasis $(13,14)$. Eya2 has been shown to be upregulated in ovarian cancer and to correlate with poor overall patient survival (15). It has also been demonstrated that Eya2 depletion inhibits the viability, growth and migration of HPV-16 transformed cervical keratinocytes (16). These studies suggested that Eya2 is a potential cancer-related protein during cancer development and progression. To date, its expression and biological roles in human astrocytoma remains unexplored, however. In this study, we examined the clinical significance of Eya2 in 90 cases of astrocytoma using immunohistochemistry. We also investigated its biological characteristics in human astrocytoma cell lines. 


\section{Materials and methods}

Patients and specimens. The study protocol was approved by the Institutional Review Board of the First Affiliated Hospital of China Medical University. A total of 90 cases of archived primary tumor specimens embedded in paraffin were obtained from the Department of Pathology. For 15 of the 90 patients, paraffin-embedded adjacent non-cancerous normal brain tissues were also obtained. These specimens were from 90 patients diagnosed with astrocytoma who underwent resection at the First Affiliated Hospital of China Medical University between 2012 and 2014. Informed consent was obtained from all these patients. Histological diagnosis was performed and the astrocytoma tissues were classified as grades I-IV according to the WHO classification guidelines (2007).

Immunohistochemistry. Surgically excised astrocytoma specimens were fixed in $10 \%$ neutral buffered formalin, embedded in paraffin, and $4-\mu \mathrm{m}$-thick sections were prepared. Astrocytoma tissue sections were used for immunostaining with the Maixin Elivision plus kit (Fuzhou Maixin Biotechnology Co., Ltd., Fuzhou, China). The sections were deparaffinized and antigen retrieval was performed in citrate buffer for 2 min using an autoclave. Endogenous peroxidase was then blocked using hydrogen peroxide. Eya2 rabbit polyclonal antibody (1:300 dilution; 11314-1-AP; Proteintech Inc., Chicago, IL, USA) was used to incubate the tissue sections at $4^{\circ} \mathrm{C}$ overnight. Incubation with polymer secondary antibody was performed after washing with phosphate-buffered saline (PBS). The DAB plus kit (Fuzhou Maixin Biotechnology Co., Ltd.) was used to develop the staining. Counterstaining was carried out using hematoxylin, and the sections were dehydrated in alcohol before mounting.

Strong nuclear staining was regarded as positive. Samples with $<15 \%$ strong nuclear staining were regarded as having a negative/low expression. Tumor samples with $\geq 15 \%$ strong nuclear staining were considered to have a Eya2 high expression.

Cell culture and transfection. The U251, U87, A172 cell lines were obtained from the American Type Culture Collection (ATCC; Manassas, VA, USA). The cells were cultured in Dulbecco's modified Eagle's medium (DMEM) supplemented with $10 \%$ fetal bovine serum (FBS) (HyClone, Logan, UT, USA). The astrocytoma cells were cultured in sterilized bottles. the Eya2 plasmid was obtained from OrigGene Technologies, Inc. (Rockville, MD, USA) and transfection was performed using Lipofectamine 3000 reagent. siRNA against Eya2 (Eya2 siRNA), Six1 siRNA and control siRNA were all purchased from Dharmacon (GE Healthcare Dharmacon Inc., Lafayette, CO, USA). DharmaFECT reagent (GE Healthcare Dharmacon Inc.) was used to transfect the siRNA. ERK inhibitor (PD98059) was purchased from Sigma-Aldrich (St. Louis, MO, USA). The cells were treated with PD98059 at the concentration of $20 \mu \mathrm{M}$ for $6 \mathrm{~h}$.

Reverse transcription-quantitative PCR (RT-qPCR). RNA was isolated from the cells and tissues using TRIzol reagent (Life Technology, St. Louis, MO, USA) according to the manufacturer's instructions. cDNA was obtained using the PrimerScript RT Master Mix kit (Takara, Dalian, China). $20 \mu 1$ of reverse-transcription reaction solution consisted of
$4 \mu 15 \mathrm{X}$ RT Master Mix and $800 \mathrm{ng}$ RNA. The reaction procedure was $85^{\circ} \mathrm{C}, 2 \mathrm{~min}$ and $37^{\circ} \mathrm{C}, 30 \mathrm{~min}$. Quantitative PCR was performed using SYBR-Green PCR Master mix (Takara Bio, Inc., Shiga, Japan) in a total volume of $20 \mu 1$ on 7900 Real-Time PCR system (Applied Biosystems, Foster City, CA, USA) as follows: $95^{\circ} \mathrm{C}$ for $30 \mathrm{sec}, 40$ cycles of $95^{\circ} \mathrm{C}$ for $5 \mathrm{sec}, 60^{\circ} \mathrm{C}$ for $30 \mathrm{sec} . \beta$-actin was used as the reference gene. The relative levels of gene expression were calculated using the $2^{-\Delta \Delta C q}$ method. The sequences of the primers are listed as follows: Eya2 forward, 5'-CACTCCCTGAAGGCACTAAACCTC ATC-3' and reverse, 5'-CTGCATTATCCTCTCGAAGCAGCT CTC-3'; cyclin D1 forward, 5'-TGGAGGTCTGCGAGGA ACA-3' and reverse, 5'-TTCATCTTAGAGGCCACG AACAT3'; cyclin E forward, 5'-AGCCAGCCTTGGGACAATAAT-3' and reverse, 5'-GAGCCTCTGGATGGTGCAAT-3'; MMP9 forward, 5'-CCTCTGGAGGTTCGACGTGA-3' and reverse, 5'-TAGGCTTTCTCTCGGTACTGGAA-3'; actin forward, 5'-ATAGCACAGCCTGGATAGCAACGTAC-3' and reverse, 5'-CACCTTCTACAATGAGCTGCGTGTG-3'.

Matrigel invasion assay. Cell invasion assay was performed using a Transwell chamber. The inserts were coated with $18 \mu \mathrm{l}$ Matrigel from BD Biosciences (San Jose, CA, USA) with a dilution rate of 1:4. At $48 \mathrm{~h}$ after siRNA transfection, the cells were trypsinized and $100 \mu \mathrm{l}$ of serum-free DMEM were used to dilute cells, which was added to the upper chamber followed by incubation for a further $20 \mathrm{~h}$. DMEM with $15 \%$ FBS was added to the lower chamber. The cells that passed through the filter were fixed, stained with hematoxylin and counted under a microscope (BX53; Olympus, Tokyo, Japan).

Colony formation assay and cell counting kit-8 (CCK-8) cell viability assay. For colony formation assay, following transfection, the cells were plated into $6-\mathrm{cm}$ dishes and cultured for 2 weeks. The cells were then stained with Giemsa (Sigma-Aldrich), to observe the colonies. The colonies with $>50$ cells were counted using a microscope (CX23; Olympus).

For CCK-8 assay, the cells were seeded in 96-well plates and $20 \mu \mathrm{l}$ CCK-8 solution were added to each well. CCK-8 solution was incubated with the cells for $4 \mathrm{~h}$, and the medium was then removed, and the remaining formazan was dissolved using DMSO and measured at $450 \mathrm{~nm}$ using a microplate reader (5119100, Multiskan ${ }^{\text {TM }}$ FC; Thermo Fisher Scientific, Inc., Carlsbad, CA, USA).

Western blot analysis. Total proteins were extracted using Pierce cell lysis buffer (Pierce, Thermo Fisher Scientific, Inc., Rockford, IL, USA). The proteins were quantified by the Bradford method. A total of $50 \mu \mathrm{g}$ of protein was transferred onto PVDF membranes following separation by SDS-PAGE. The membranes were incubated at $4^{\circ} \mathrm{C}$ overnight with primary antibodies against Eya2 (1:1,000; 11314-1-AP; Proteintech), Six1 (1:1,000; SAB2104425; Sigma-Aldrich), cyclin D1 (2978), cyclin E (20808), p-ERK (9101), ERK (9102), MMP9 (3852) and GAPDH (2118) (1:1,000; all from Cell Signaling Technology, Inc., Boston, MA, USA). Following incubation with HRP-coupled anti-mouse (sc-2789)/rabbit (sc-2357) IgG (1:1,000; Santa Cruz Biotechnology, Inc., Santa Cruz, CA, USA) at $37^{\circ} \mathrm{C}$ for $2 \mathrm{~h}$, the bound proteins were visualized using an ECL kit (Pierce) and detected using a DNR Bio-Imaging System (DNR Bio-Imaging 

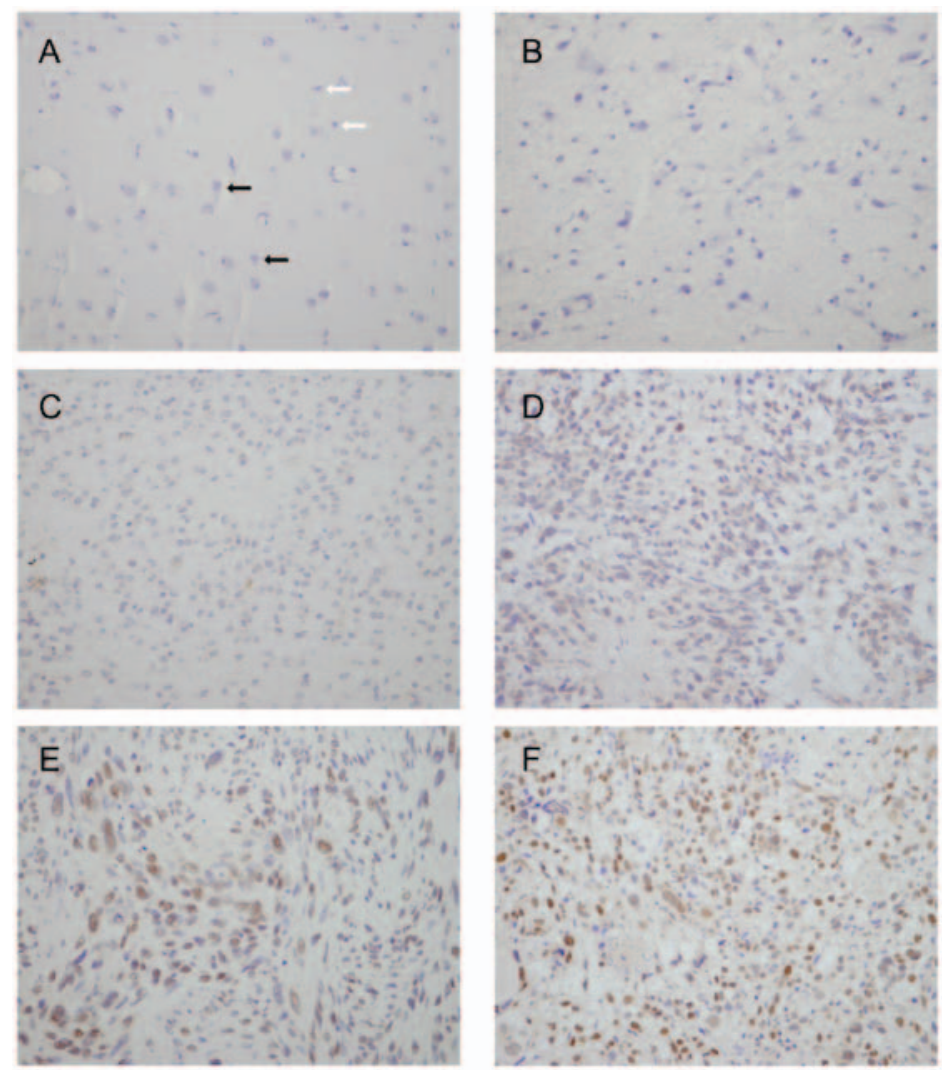

Figure 1. Expression pattern of eyes absent (Eya) 2 in human astrocytoma. (A) Immunohistochemical staining of Eya2 protein in normal brain tissue was negative in glial cells and weak in neurons. The black arrows indicate neurons and the white arrows indicate glial cells. (B) Negative Eya2 staining in pilocytic astrocytoma (grade I). (C) Negative Eya2 staining in grade II diffuse astrocytoma. (D) Weak nuclear Eya2 staining in grade III anaplastic astrocytoma. (E) Strong nuclear Eya2 staining in grade III anaplastic astrocytoma. (F) Strong nuclear Eya2 staining in glioblastoma (grade IV) (magnification, $\mathrm{x} 200$ ). Scale bar, $50 \mu \mathrm{m}$.

Systems, Ltd., Jerusalem, Israel). The densitometry of western bands was measured using Image J software.

Immunoprecipitation. A sufficient amount of antibody was added to $500 \mu \mathrm{g}$ protein and gently rotated at $4^{\circ} \mathrm{C}$ overnight. The immunocomplex was captured by adding $25 \mu$ protein A/G agarose beads (Cell Signaling Technology, Inc.) and rotated at $4^{\circ} \mathrm{C}$ for 2 to $3 \mathrm{~h}$. The mixture was centrifuged at $1,500 \mathrm{x} g$ for $5 \mathrm{~min}$ and the precipitate was washed 3 times with cold PBS, resuspended in sample buffer and boiled for $5 \mathrm{~min}$ to dissociate the immunocomplex from the beads. The supernatant was then collected by centrifugation $(1,500 \mathrm{x} g$ for 5 min at $4^{\circ} \mathrm{C}$ ) and subjected to western blot analysis.

Statistical analysis. SPSS version 16.0 software (SPSS Inc., Chicago, IL, USA) for Windows was used for all statistical analyses. The Chi-square test was used to investigate the clinical data. A t-test was used to compare other data. A value of $\mathrm{p}<0.05$ was considered to indicate a statistically significant difference. The experiments were repeated 3 times.

\section{Results}

Eya2 is upregulated in human astrocytoma tissues and correlates with tumor grade. We examined Eya2 protein expression in 90 cases of astrocytoma tissues and 15 adjacent non-cancerous tissues using immunohistochemistry. We found a negative/low expression of Eya2 in glial cells in normal
Table I. The association between eyes absent 2 (Eya 2) and the clinical characteristics of astrocytoma.

\begin{tabular}{lcccc}
\hline & & \multicolumn{2}{c}{ Eya2 expression } & \\
\cline { 3 - 4 } Parameters & Patients & Low & High & P-value \\
\hline Age (years) & & & & 0.62 \\
$<50$ & 68 & 42 & $26(38.2 \%)$ & \\
$\geq 50$ & 22 & 15 & $7(31.8 \%)$ & \\
Gender & & & & 0.82 \\
Male & 58 & 36 & $22(37.9 \%)$ & \\
Female & 32 & 21 & $11(34.3 \%)$ & \\
Grades & & & & 0.03 \\
I & 8 & 8 & $0(0 \%)$ & \\
II & 30 & 22 & $8(26.7 \%)$ & \\
III & 22 & 12 & $10(45.5 \%)$ & \\
IV & 30 & 15 & $15(50 \%)$ & \\
& & & &
\end{tabular}

tissue (Fig. 1A). Positive nuclear Eya2 staining was observed in $36.7 \%$ (33/90) of the astrocytoma tissues (Fig. 1B-F). We also analyzed the correlation between Eya2 expression and clinical characteristics. A high Eya2 expression was found to positively correlate with an advanced tumor grade. As shown in Table I, 
A

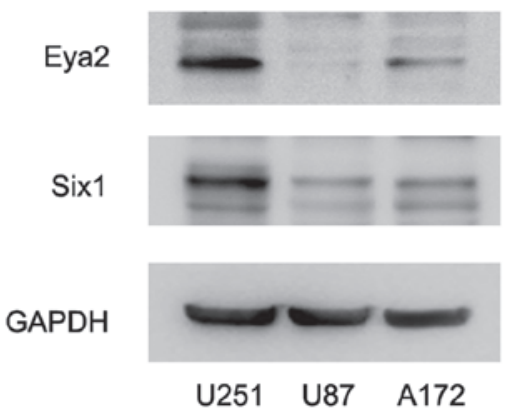

B
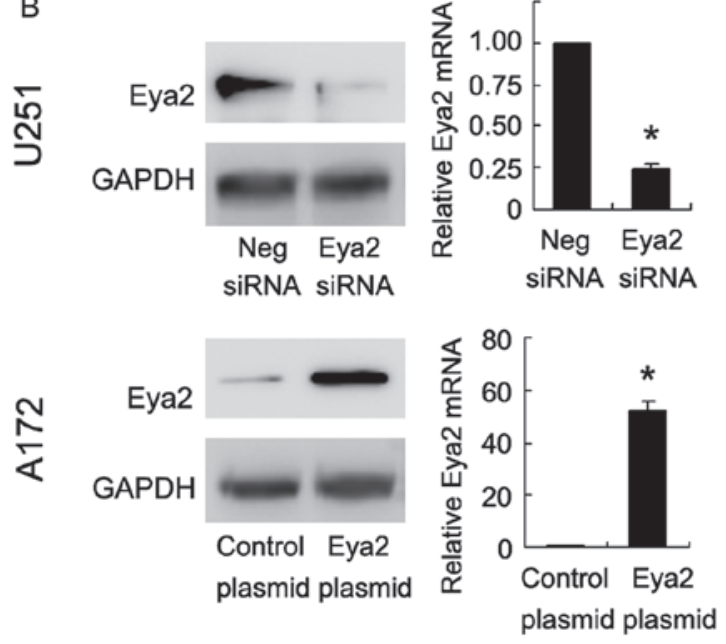

Figure 2. Eyes absent (Eya) 2 expression in astrocytoma cell lines. (A) Western blot analysis indicated that Eya2 and Six1 protein expression was high in the U251 cell line and low in the U87 and A172 cell lines. (B) Eya2 siRNA treatment decreased its mRNA and protein expression in U251 cells. ${ }^{*} \mathrm{P}<0.05$ Eya 2 siRNA vs Neg siRNA in U251 cells. Eya2 plasmid transfection upregulated its mRNA and protein levels. ${ }^{*} \mathrm{P}<0.05$ Eya2 plasmid vs control plasmid in A172 cells.

the positive rates of Eya2 protein in grade I (pilocytic astrocytoma), grade II (diffuse astrocytoma), grade III (anaplastic astrocytoma) and grade IV (glioblastoma) astrocytoma were $0 \%(0 / 8), 26.7 \%(8 / 30), 45.5 \%(10 / 22)$ and $50 \%(15 / 30)$, respectively $(\mathrm{p}=0.03)$. No correlation was found between Eya2 and age $(\mathrm{p}=0.62)$ and gender $(\mathrm{p}=0.82)$.

Eya2 regulates the proliferation andinvasion of astrocytoma cell lines. In order to explore the biological function of Eya2 in astrocytoma cells, we analyzed its expression level in 3 astrocytoma cell lines (U251, U87 and A172) (Fig. 2A). Transfection with the Eya2 expression plasmid was performed in the A172 cell line, which was fouond to have a low endogenous expression of Eya2. siRNA knockdown was carried out in the U251 cell line, which was found to have a high expression of Eya2 (Fig. 1A). As shown in Fig. 2B, the protein and mRNA levels of Eya2 were significantly increased following transfection with the expression plasmid, and were decreased following transfection with siRNA.

The role of Eya2 in cell growth was examined by CCK-8 cell viability assay (Fig. 3A). The results revealed that transfection with the Eya2 expression plasmid increased the cell growth rate and the silencing of Eya2 inhibited cell proliferation. Colony formation assay revealed that transfection with the Eya2 expression plasmid significantly increased the colony number in the A172 cells. On the contrary, the knockdown of Eya2 in the
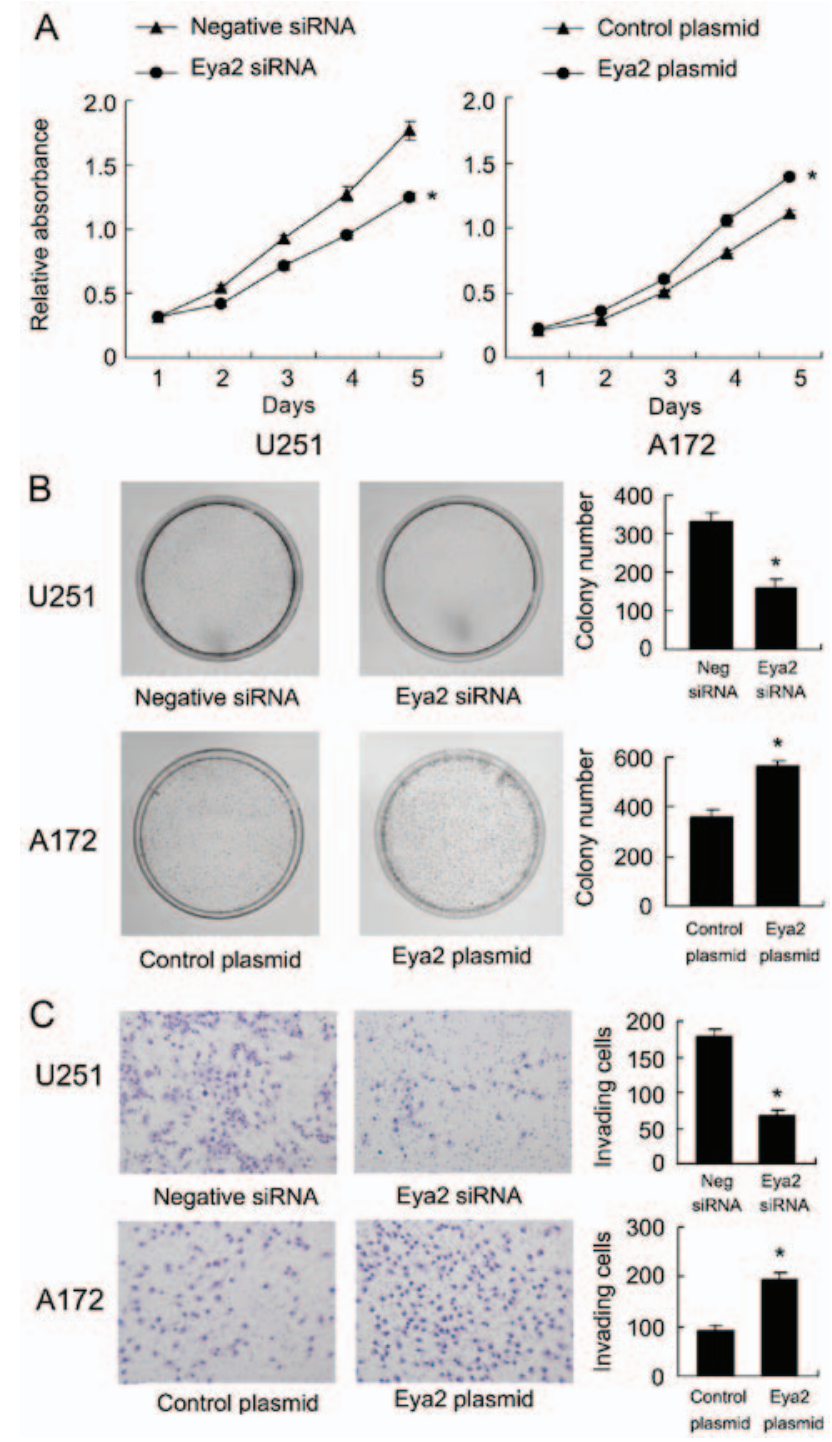

Figure 3. Eyes absent (Eya) 2 regulates the growth and invasion of astrocytoma cells. (A) Cell Counting kit-8 (CCK-8) assay demonstrated that Eya2 overexpression accelerated cell growth rate, while Eya2 knockdown inhibited cell proliferation. (B) Colony formation assay demonstrated that Eya2 overexpression upregulated the colony number, while Eya2 knockdown downregulated the colony number. ${ }^{\text {}} \mathrm{P}<0.05$ Eya2 siRNA vs Neg siRNA in U251 cells. ${ }^{*} \mathrm{P}<0.05$ Eya2 plasmid vs control plasmid in A172 cells. (C) Matrigel invasion assay revealed that Eya2 knockdown decreased cell invasion in the U251 cell line. "P $<0.05$ Eya2 siRNA vs Neg siRNA in U251 cells. Transfection with Eya2 expession plasmid increased the A172 invading cell number. ${ }^{*} \mathrm{P}<0.05$ Eya2 plasmid vs control plasmid in A172 cells.

U251 cells significantly decreased the colony number (Fig. 3B). Transwell invasion assay was also performed to examine the role of Eya 2 in the invasion of both the A172 and U251 cell lines. The results revealed that the knockdown of Eya2 decreased the number of invading U251 cells, and that Eya2 overexpression in the A172 cells facilitated cell invasion (Fig. 3C).

Eya 2 regulates cell cycle progression and related protein expression. The above-mentioned results indicated that Eya2 promoted astrocytoma cell proliferation and invasion. Thus, we further examined the effect of Eya 2 on cell cycle progression. As shown in Fig. 4A, Eya2 overexpression in the A172 cells facilitated the G1/S transition, while the knockdown of Eya2 in the U251 cells decreased the percentage of 
A

251

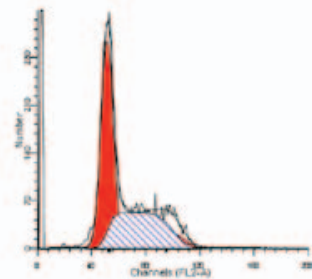

Negative siRNA

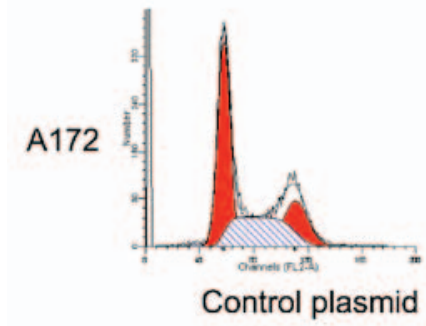

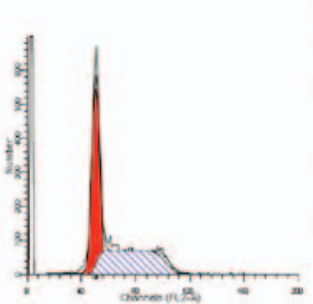

Eya2 siRNA

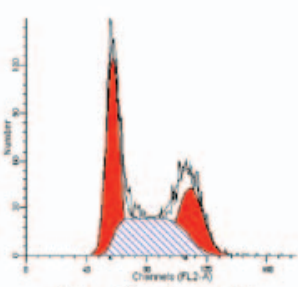

Eya2 plasmid

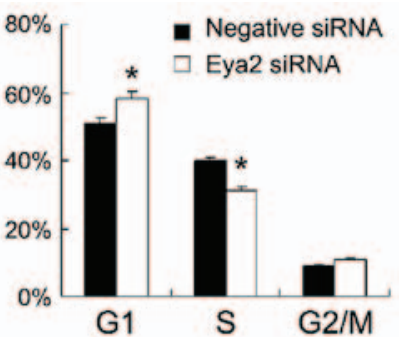

${ }^{80 \%}[\quad$ Control plasmid $\square$ Eya2 plasmid

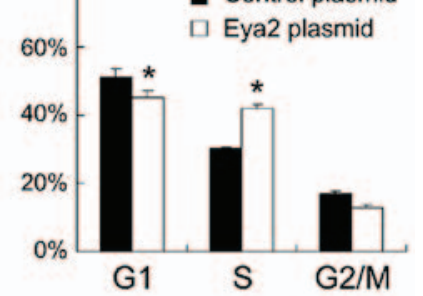

B

cyclin D1

cyclin $\mathrm{E}$

MMP9

p-ERK

ERK

GAPDH

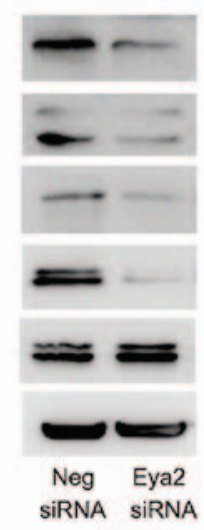

U251

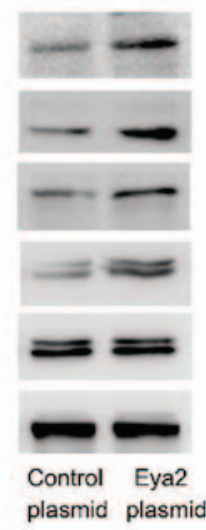

A172
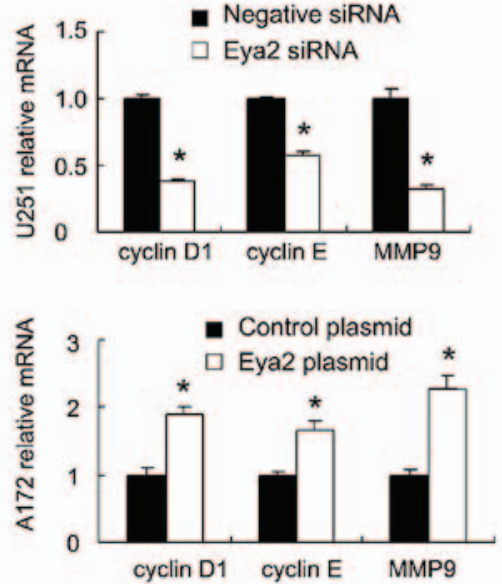

Figure 4. Eyes absent (Eya) 2 regulates cell cycle and related protein expression. (A) Eya2 overexpression in A172 cells increased the S phase percentage and decreased G1 phase percentage. Eya2 knockdown by siRNA in U251 cells had the opposite effect. "P $<0.05$ Eya2 siRNA vs Neg siRNA in U251 cells. "P $<0.05$ Eya2 plasmid vs control plasmid in A172 cells. (B) Eya2 overexpression upregulated cyclin D1, cyclin E, MMP9 mRNA and protein expression in the A172 cell line. "P<0.05 Eya2 plasmid vs control plasmid in A172 cells. Eya2 knockdown downregulated the expression of these proteins in the U251 cells. "P<0.05 Eya2 siRNA vs Neg siRNA in U251 cells. Eya2 overexpression activated ERK phosphorylation, while Eya2 knockdown downregulated ERK phosphorylation.

cells in the $\mathrm{S}$ phase. We further examined the levels of cell cycle-related proteins and found that Eya2 overexpression positively regulated cyclin $\mathrm{D} 1$ and cyclin $\mathrm{E}$ mRNA and protein expression (Fig. 4B). However, the knockdown of Eya2 in the U251 cell line downregulated the mRNA and protein expression of cyclin D1 and cyclin E. In addition, Eya2 overexpression upregulated the mRNA and protein levels of MMP9, a protein that is closely associated with cancer invasiveness (Fig. 4B).

Eya 2 regulates cell invasion through ERK signaling. To explore the potential mechanisms of the Eya2 regulation of cell invasion, we examined the changes in several signaling pathways previously reported to be involved in cancer invasion (17-21). We found that Eya2 overexpression upregulated ERK phosphorylation, while Eya2 knockdown downregulated ERK phosphorylation (Fig. 4B), suggesting that the ERK pathway may be responsible for the invasion-promoting effects of Eya2. To confirm the role of ERK as the mediator of invasiveness, the ERK inhibitor, PD98059 (20 $\mu \mathrm{M}, 6 \mathrm{~h})$, was used to treat the A172 cells transfected with the Eya2 expression plasmid. As shown in Fig. 5A, treatment with the ERK inhibitor blocked the promoting effect of Eya2 on cell invasion. In addition, western blot analysis and PCR revealed that treatment with the ERK inhibitor abolished the effect of Eya2 on MMP9 upregulation in the A712 cell line at both the mRNA and protein level (Fig. 5B).

Eya 2 interacts with Sixl protein in U251 cells. Eya2 has been previously regarded as a Six 1 partner, which has been reported to be an important cancer-related protein $(22,29)$. To confirm this, immunoprecipitation was performed. We immunoprecipitated Eya2 from U251 cell lysate and analyzed it for Six1 binding. As shown in Fig. 5C, Eya2 co-immunoprecipitated with Six1 in the U251 cells. We also examined the protein expression of Six1 in 3 astrocytoma cell lines. We found that Six1 expression was high in the U251 cells and low in the U87 and A172 cells, which closely correlated with the Eya2 levels (Fig. 2A). To validate the involvement of Six1 in the biological role of Eya2, we adopted Six1 siRNA to deplete its endogenous expression in the A172 cells (Fig. 5D). Western blot analysis and real-time PCR analysis demonstrated that the depletion of Six1 downregulated MMP9 expression in the A172 cells. In addition, Eya2 overexpression failed to upregulated MMP9 mRNA and 
A

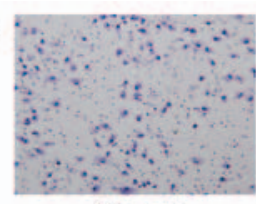

Control

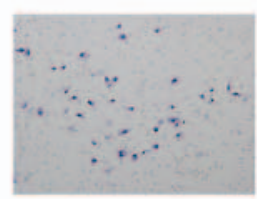

Control+PD98059

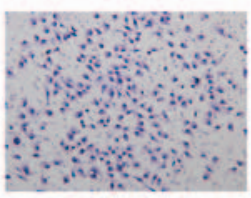

Eya2 plasmid

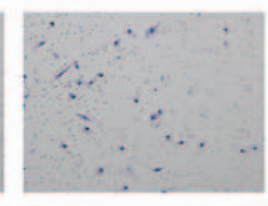

Eya2 plasmid+PD98059

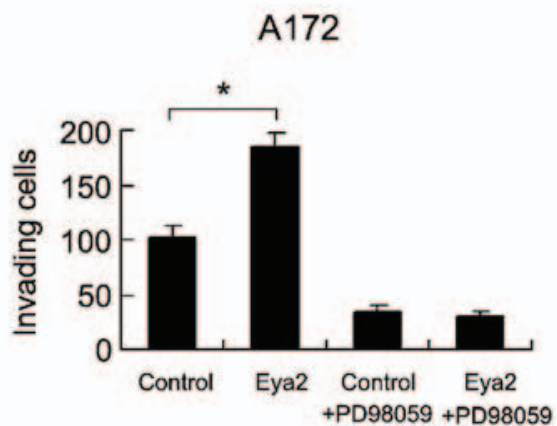

B

A172

MMP9

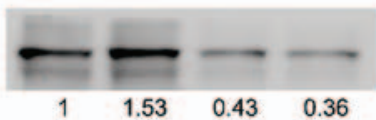

GAPDH
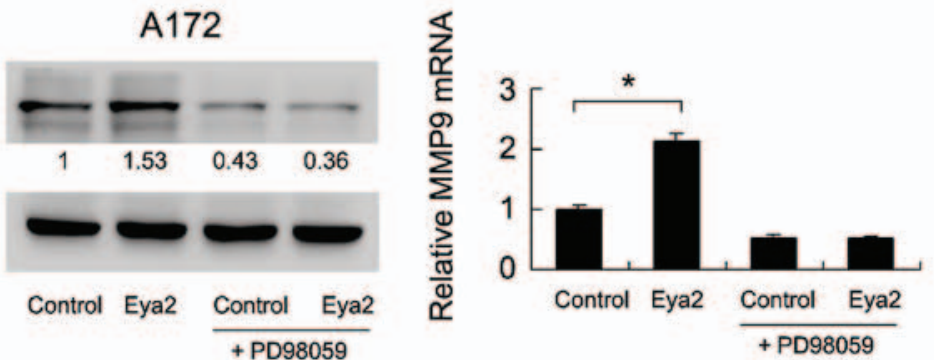

C Six1

Eya2
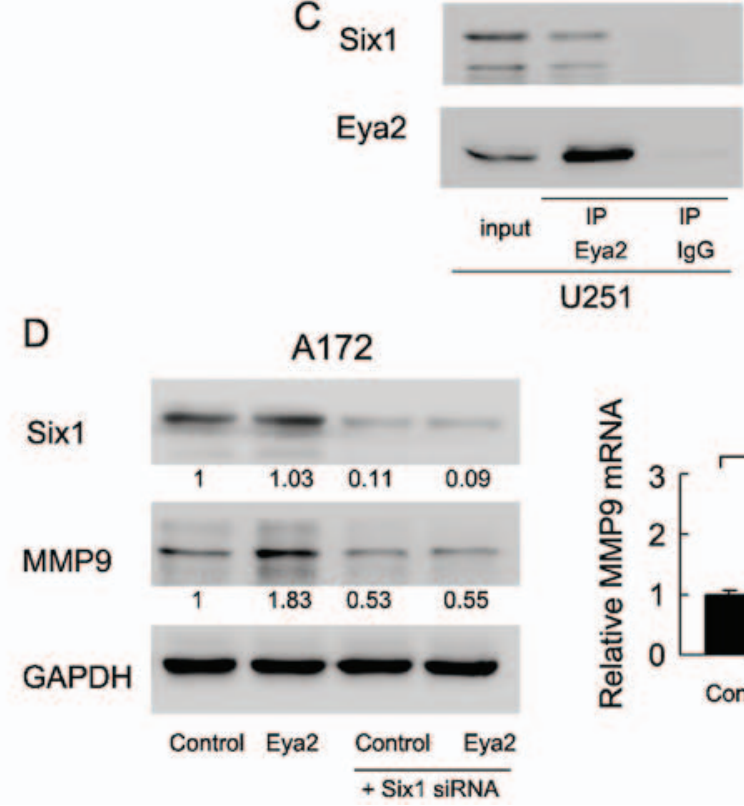

D

A172

U251

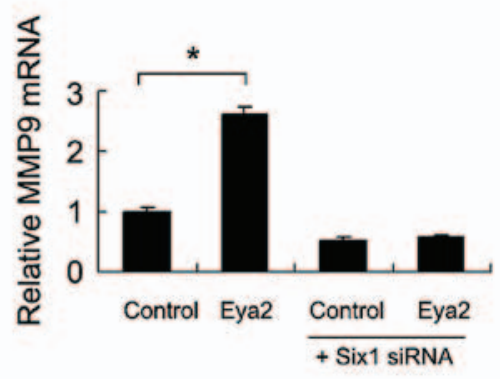

Figure 5. Eyes absent (Eya) 2 interacts with Six 1 and regulates ERK signaling. (A) ERK inhibitor, PD98059, blocked the promoting effect of Eya2 on A172 invasion. ${ }^{*} \mathrm{P}<0.05$ Eya2 plasmid vs control plasmid in A172 cells. (B) Western blot analysis revealed that ERK inhibitor, PD98059, abolished the promoting effect of Eya2 on MMP9 mRNA and protein expression. " $\mathrm{P}<0.05$ Eya2 plasmid vs control plasmid in A172 cells. (C) Eya2 co-immunoprecipitated with Six1 in U251 cells. (D) Transfection with Six1 siRNA significantly downregulated its protein in A172 cells. In Six1-depleted cells, transfection with Eya2 expression plasmid could not upregulate MMP9 expression at both the mRNA and protein level. ${ }^{*} \mathrm{P}<0.05$ Eya2 plasmid vs control plasmid in A172 cells. Densitometry of western bands was measured using ImageJ software and relative intensity was calculated and indicated.

protein in the Six1-depleted cells, which indicated Six1 that is required for the biological effects of Eya2.

\section{Discussion}

Eya2 overexpression has been reported in several human malignancies $(15,16,23)$. In this study, we found that Eya2 protein was overexpressed in $36.7 \%$ of human astrocytoma specimens, and its expression was higher in grade III and IV astrocytomas than in grade I and II astrocytomas. Using siRNA and plasmid transfection, we demonstrated that the knockdown of Eya2 significantly inhibited the U251 cell growth rate and colony formation ability. Matrigel invasion assay revealed that Eya2 overexpression promoted the invasive ability, while Eya2 knockdown inhibited the invasive ability. Since Eya2 overexpression accelerated and its depletion inhibited astrocytoma cell growth and colony formation, we examined the changes in cell cycle progression. In accordance with the above data, Eya2 facilitated cell cycle transition, particularly during the G1/S checkpoint. We also found that Eya2 overexpression upregulated the level of cyclin D1 and cyclin E, which are key regulators of cell cycle progression, particularly during the G1/S checkpoint. Previous studies have demonstrated that cyclin D1/E are important cancer markers during astrocytoma progression $(24,25)$. Thus, Eya2 
promotes astrocytoma cell proliferation possibly through the regulation of cell cycle-related proteins. It was has been reported that Eya2 is required to mediate the pro-metastatic functions of Six1 via the induction of transforming growth factor (TGF)- $\beta$ signaling (14). Eya2 also serves as an oncogene in cervical carcinogenesis by promoting viability, migratory capacity and anchorage-independent growth (16). Eya2 also serves as an oncogene in breast cancer cells (23). Our biological results are in accordance with those of the above-mentioned previous studies indicating Eya2 is an oncogene which can regulate both the proliferation and invasion of astrocytoma cells.

The molecular mechanisms of action of Eya2 related to the promotion of cell invasion were also investigated by examining related proteins and signaling pathways. We found that Eya2 overexpression positively regulated ERK signaling, and regulated MMP9 mRNA and protein expression. ERK activation has been demonstrated to facilitate astrocytoma invasion and metastasis via the upregulation of MMP family proteins (26-28). Importantly, treatment with the ERK inhibitor, PD98059, abolished the promoting effects of Eya2 on both cell invasion and MMP9 protein expression. These results indicated that Eya2 promoted astrocytoma invasion possibly through ERK signaling.

Eya2 has been reported as the binding partner of Six1, which serves as an oncoprotein in multiple types of cancer, including astrocytoma (29-32). Six1 and Eya2 have been reported to be critical for metastasis in a breast cancer model $(14,29)$. Six1 has also been reported to promote cancer proliferation and invasion through the regulation of cell cycle protein and ERK signaling $(30,33,34)$. Thus, we hypothesized that the effects of Eya2 on ERK signaling and MMP9 are dependent on its association with Six1. To validate this hypothesis, we first confirmed that Eya2 can interact with Six1 protein using immunoprecipitation. Second, we found that Six1 protein levels closely correlated with Eya2 expression in the cell lines. Third, the depletion of Six 1 by siRNA blocked the promoting effect of Eya2 on MMP9 expression. Taken together, these results suggest that Eya2 exerts its oncogenic effects by interacting with Six1 protein and activating ERK/MMP9 signaling.

In conclusion, the present study demonstrated that Eya2 was overexpressed in human astrocytoma and correlated with an advanced tumor grade. Eya2 interacts with Six1 and promotes astrocytoma cell proliferation and invasion through the upregulation of cyclin proteins and ERK signaling.

\section{References}

1. Siegel R, Naishadham D and Jemal A: Cancer statistics, 2012. CA Cancer J Clin 62: 10-29, 2012.

2. Krupkova O Jr, Loja T, Redova M, Neradil J, Zitterbart K, Sterba J and Veselska R: Analysis of nuclear nestin localization in cell lines derived from neurogenic tumors. Tumour Biol 32: 631-639, 2011.

3. Zadran S, Amighi A, Otiniano E, Wong K and Zadran $\mathrm{H}$ : ENTPD5-mediated modulation of ATP results in altered metabolism and decreased survival in gliomablastoma multiforme. Tumour Biol 33: 2411-2421, 2012.

4. Chi F, Wu R, Jin X, Jiang M and Zhu X: HER2 induces cell proliferation and invasion of non-small-cell lung cancer by upregulating COX-2 expression via MEK/ERK signaling pathway. Onco Targets Ther 9: 2709-2716, 2016.

5. Jiang Q, Pan Y, Cheng Y, Li H, Liu D and Li H: Lunasin suppresses the migration and invasion of breast cancer cells by inhibiting matrix metalloproteinase-2/-9 via the FAK/Akt/ERK and NF-кB signaling pathways. Oncol Rep 36: 253-262, 2016.
6. Ko HS, Park BJ, Choi SK, Kang HK, Kim A, Kim HS, Park IY and Shin JC: STAT3 and ERK signaling pathways are implicated in the invasion activity by oncostatin $\mathrm{M}$ through induction of matrix metalloproteinases 2 and 9. Yonsei Med J 57: 761-768, 2016.

7. Dong DD, Zhou H and Li G: ADAM15 targets MMP9 activity to promote lung cancer cell invasion. Oncol Rep 34: 2451-2460, 2015.

8. Kalhori V and Törnquist K: MMP2 and MMP9 participate in S1P-induced invasion of follicular ML-1 thyroid cancer cells. Mol Cell Endocrinol 404: 113-122, 2015.

9. Jacob A, Jing J, Lee J, Schedin P, Gilbert SM, Peden AA, Junutula JR and Prekeris R: Rab40b regulates trafficking of MMP2 and MMP9 during invadopodia formation and invasion of breast cancer cells. J Cell Sci 126: 4647-4658, 2013.

10. Silver SJ, Davies EL, Doyon L and Rebay I: Functional dissection of eyes absent reveals new modes of regulation within the retinal determination gene network. Mol Cell Biol 23: 5989-5999, 2003.

11. Kumar JP and Moses K: EGF receptor and Notch signaling act upstream of Eyeless/Pax6 to control eye specification. Cell 104: 687-697, 2001.

12. Kenyon KL, Ranade SS, Curtiss J, Mlodzik M and Pignoni F: Coordinating proliferation and tissue specification to promote regional identity in the Drosophila head. Dev Cell 5: 403-414, 2003.

13. Krishnan N, Jeong DG, Jung SK, Ryu SE, Xiao A, Allis CD, Kim SJ and Tonks NK: Dephosphorylation of the C-terminal tyrosyl residue of the DNA damage-related histone H2A.X is mediated by the protein phosphatase eyes absent. J Biol Chem 284: 16066-16070, 2009.

14. Farabaugh SM, Micalizzi DS, Jedlicka P, Zhao R and Ford HL: Eya2 is required to mediate the pro-metastatic functions of Six1 via the induction of TGF- $\beta$ signaling, epithelial-mesenchymal transition, and cancer stem cell properties. Oncogene 31: 552-562, 2012.

15. Zhang L, Yang N, Huang J, Buckanovich RJ, Liang S, Barchetti A, Vezzani C, O'Brien-Jenkins A, Wang J, Ward MR, et al: Transcriptional coactivator Drosophila eyes absent homologue 2 is up-regulated in epithelial ovarian cancer and promotes tumor growth. Cancer Res 65: 925-932, 2005.

16. Bierkens M, Krijgsman O, Wilting SM, Bosch L, Jaspers A, Meijer GA, Meijer CJ, Snijders PJ, Ylstra B and Steenbergen RD: Focal aberrations indicate EYA2 and hsa-miR-375 as oncogene and tumor suppressor in cervical carcinogenesis. Genes Chromosomes Cancer 52: 56-68, 2013.

17. Bai G, Chu J, Eli M, Bao Y and Wen H: PAQR3 overexpression suppresses the aggressive phenotype of esophageal squamous cell carcinoma cells via inhibition of ERK signaling. Biomed Pharmacother 94: 813-819, 2017.

18. Li J, Chen S and Ni B: Upregulation of HINT2 inhibits non-small cell lung cancer cell invasion via $\mathrm{COX}-2 / \mathrm{PGE}_{2}$-mediated activation of $\beta$-catenin signaling. Oncol Res: Aug 11, 2017 (Epub ahead of print).

19. Song J, Guan Z, Li M, Sha S, Song C, Gao Z and Zhao Y: MicroRNA-154 inhibits the growth and invasion of gastric cancer cells by targeting DIXDC1/WNT signaling. Oncol Res: Aug 11, 2017 (Epub ahead of print).

20. Liao Y, Yuan S, Chen X, Zhu P, Li J, Qin L and Liao W: Up-regulation of BRCA1-associated RING domain 1 promotes hepatocellular carcinoma progression by targeting Akt signaling. Sci Rep 7: 7649, 2017.

21. Jin H, Xie Q, Guo X, Xu J, Wang A, Li J, Zhu J, Wu XR, Huang H and Huang $\mathrm{C}: \mathrm{p} 63 \alpha$ protein upregulates heat shock protein 70 expression via E2F1 transcription factor 1, promoting Wasf3/ Wave3/MMP9 signaling and bladder cancer invasion. J Biol Chem: Aug 9, 2017 (Epub ahead of print).

22. Patrick AN, Cabrera JH, Smith AL, Chen XS, Ford HL and Zhao R: Structure-function analyses of the human SIX1-EYA2 complex reveal insights into metastasis and BOR syndrome. Nat Struct Mol Biol 20: 447-453, 2013.

23. Fu J, Xu X, Kang L, Zhou L, Wang S, Lu J, Cheng L, Fan Z, Yuan B, Tian P, et al: miR-30a suppresses breast cancer cell proliferation and migration by targeting Eya2. Biochem Biophys Res Commun 445: 314-319, 2014.

24. Abdullah JM, Ahmad F, Ahmad KA, Ghazali MM, Jaafar H, Ideris A, Ali AM, Omar AR, Yusoff K, Lila MA, et al: Molecular genetic analysis of BAX and cyclin D1 genes in patients with malignant glioma. Neurol Res 29: 239-242, 2007.

25. Arato-Ohshima T1 and Sawa H: Over-expression of cyclin D1 induces glioma invasion by increasing matrix metalloproteinase activity and cell motility. Int J Cancer 83: 387-392, 1999. 
26. Velpula KK, Rehman AA, Chelluboina B, Dasari VR, Gondi CS, Rao JS and Veeravalli KK: Glioma stem cell invasion through regulation of the interconnected ERK, integrin $\alpha 6$ and N-cadherin signaling pathway. Cell Signal 24: 2076-2084, 2012.

27. Das G, Shiras A, Shanmuganandam K and Shastry P: Rictor regulates MMP-9 activity and invasion through Raf-1-MEK-ERK signaling pathway in glioma cells. Mol Carcinog 50: 412-423, 2011

28. Lakka SS, Jasti SL, Gondi C, Boyd D, Chandrasekar N, Dinh DH, Olivero WC, Gujrati M and Rao JS: Downregulation of MMP-9 in ERK-mutated stable transfectants inhibits glioma invasion in vitro. Oncogene 21: 5601-5608, 2002.

29. Blevins MA, Towers CG, Patrick AN, Zhao R and Ford HL: The SIX1-EYA transcriptional complex as a therapeutic target in cancer. Expert Opin Ther Targets 19: 213-225, 2015.

30. Li Z, Tian T, Lv F, Chang Y, Wang X, Zhang L, Li X, Li L, Ma W, Wu J, et al: Six 1 promotes proliferation of pancreatic cancer cells via upregulation of cyclin D1 expression. PLoS One 8: e59203, 2013.

31. Ono H, Imoto I, Kozaki K, Tsuda H, Matsui T, Kurasawa Y, Muramatsu T, Sugihara K and Inazawa J: SIX1 promotes epithelial-mesenchymal transition in colorectal cancer through ZEB1 activation. Oncogene 31: 4923-4934, 2012.
32. Tian T, Li A, Lu H, Luo R, Zhang M and Li Z: Six1 promotes glioblastoma cell proliferation and invasion by upregulation of connective tissue growth factor. Am J Cancer Res 5: 1823-1830, 2015.

33. Iwanaga R, Wang CA, Micalizzi DS, Harrell JC, Jedlicka P, Sartorius CA, Kabos P, Farabaugh SM, Bradford AP and Ford HL: Expression of Six1 in luminal breast cancers predicts poor prognosis and promotes increases in tumor initiating cells by activation of extracellular signal-regulated kinase and transforming growth factor-beta signaling pathways. Breast Cancer Res 14: R100, 2012.

34. Coletta RD, Christensen K, Reichenberger KJ, Lamb J, Micomonaco D, Huang L, Wolf DM, Müller-Tidow C, Golub TR, Kawakami K, et al: The Six1 homeoprotein stimulates tumorigenesis by reactivation of cyclin A1. Proc Natl Acad Sci USA 101: 6478-6483, 2004. 